recurrences were detected among the remaining patients during the follow up period.

Conclusions: Our series suggest that recurrence can occur in extrapleural SFTs even with low risk features. The treatment of choice is complete resection followed by extended follow-up surveillance.

\section{MUSCLE PATHOLOGY: TEACHING OPPORTUNITIES AND STATEGIES IN A PATHOLOGY DEPARTMENT IN SINGAPORE}

Kong-Bing Tan, Yoke-Sun Lee

Department of Pathology, Yong Loo Lin School of Medicine, National University Health System, Singapore

Background: Muscle pathology is a highly sub-specialized discipline in histopathology. While it does not generally feature prominently in routine histopathology practice or in fellowship or board examinations, some training exposure to the discipline for histopathology trainees is often deemed desirable.

In Singapore, histopathology trainees are given exposure to the discipline through sessions in the national training programme, intradepartmental neuropathology teaching as well as opportunistically during work at pathology departments.

Implementation: During recent national training programme teaching sessions, muscle pathology was introduced via a lecture on the general approach to muscle biopsy interpretation and a separate presentation of short clinico-pathological cases with quizzes. Conditions covered include muscular dystrophy, mitochondrial myopathy, central core myopathy, glycogen storage disease, polymyositis, disuse atrophy and neurogenic atrophy. In our department, trainees undertake structured training in the major subspecialties of histopathology, including neuropathology. They receive weekly illustrative slides for preview, culminating in a multi-header teaching session with the teaching pathologist. For neuropathology, a session on muscle pathology is included.

During routine pathology work, muscle biopsies are channeled to the subspecialty pathologists for reporting. When interesting or diagnostically-typical cases are encountered, they are shared with trainees and interested colleagues.

Conclusions: Through the above teaching opportunities, trainees acquire a working knowledge of muscle pathology. From informal feedback, they appear to particularly like the casebased quiz teaching format. Many feel the discipline is relevant to their overall training in histopathology while some, if given the opportunity, may consider developing subspecialty interest in muscle pathology, auguring well for the future of the discipline.

\section{PATHOLOGY OF SURGICALLY EXCISED PRIMARY CARDIAC TUMORS: A REVIEW OF 39 YEAR SINGLE INSTITUTION EXPERIENCE}

\section{Felipe S. Templo Jr}

Division of Laboratory Medicine, Philippine Heart Center, Quezon City, Philippines

Background: Primary cardiac tumors are uncommon clinical entities, and thus, there are relatively limited descriptions in the published literature.
Aims: Most of these tumors have nonspecific presentations and thus, a clinico-pathologic review may provide additional information of potentially practical value for relevant clinical diagnosis and future translational researches.

Method: The surgical pathology files spanning a 39-year period (1975-2014) were reviewed for all surgically excised cardiac tumors. There were 353 of 357 (98.9\%) cases that were clinically established as primary.

Results: Of 353 primary cardiac tumors, 327 (92.6\%) were classified as benign, while $26(7.4 \%)$ were classified as malignant. There was female sex predilection for both benign and malignant tumors with a male to female ratio of $1: 2.8$. Of the benign tumors, the 5 most common histologic types were myxoma (95.1\%), fibroma (1.2\%), rhabdomyoma (1.2\%), hemangioma $(0.6 \%)$, and leiomyoma $(0.6 \%)$. The locations in decreasing frequency were left atrium, right atrium, left ventricle, and right ventricle. Tumor sizes ranged from 0.4 to $8.7 \mathrm{~cm}$. The average age of patient was 51.4 years. Among the malignant tumors, the 5 most common histologic types were myofibroblastic sarcoma (15.4\%), undifferentiated sarcoma (15.4\%), angiosarcoma (11.5\%), malignant fibrous histiocytoma $(11.5 \%)$ and rhabdomyosarcoma $(11.5 \%)$. Locations in decreasing frequency were left atrium, right atrium, right ventricle, ventricle and atrium, biventricular, and biatrial. Tumor sizes ranged from 0.8 to $10.6 \mathrm{~cm}$. Average age of patients was 39.8 years.

Conclusions: In this series, primary cardiac tumors generally have significant female sex predilection. Primary malignant cardiac tumors however occurred in a relatively younger age group than their benign counterparts.

\section{GENOMIC AND IMMUNOHISTOCHEMICAL PROFILES OF ENTEROPATHY-ASSOCIATED T-CELL LYMPHOMA IN JAPAN}

$\underline{\text { Sakura Tomita }^{1}}$, Yara Y. Kikuti ${ }^{1}$, Joaquim Carreras Esteban ${ }^{1}$, Katsuyoshi Takata $^{2}$, Tadashi Yoshino ${ }^{2}$, Sílvia Beà ${ }^{3}$, Elias Campo ${ }^{3}$, Naoya Nakamura

${ }^{1}$ Department of Pathology, Tokai University, School of Medicine, Isehara, ${ }^{2}$ Department of Pathology, Dentistry and Pharmaceutical Sciences, Okayama University Graduate School of Medicine, Okayama, Japan; and ${ }^{3}$ Hematopathology Unit, Hospital Clinic Barcelona, Institut d'Investigacions Biomediques August Pi i Sunyer (IDIBAPS), University of Barcelona, Barcelona, Spain

Background: Enteropathy-associated T-cell lymphoma (EATL) is a rare primary T-cell lymphoma of the digestive tract. EATL is classified as either Type I, which is frequently associated with and though to arise from celiac disease and is primarily observed in Northern Europe, and Type II, which occurs de novo and is distributed all over the world with predominance in Asia. Its pathogenesis in Asia is unknown.

Aims: We aimed to clarify the histological and genomic profiles of EATL in Japan in homogeneous series of 20 cases.

Methods: The cases were characterized by immunohistochemistry, high resolution oligonucleotide microarray, and fluorescence in situ hybridization (FISH) at 5 different loci: 1q21.3 (CKS1B), 6q16.3 (HACE1), 7p22.3 (MAFK), 9q33.3 (PPP6C) and 9q34.3 (ASS1, CARD9) using FFPE sections.

Results: The histological appearance ranged from medium- to large-sized cells in 13 cases (65\%), small-to-medium-sized cells 